\title{
Abdominal resection of a cornual heterotopic pregnancy using intraoperative ultrasound: case report
}

\author{
Abigail C. Mancuso, ${ }^{1}$ Adrianne Racek, ${ }^{1}$ E. Hakan Duran ${ }^{1}$ \\ Keywords: heterotopic pregnancy, cornual ectopic, in vitro fertilization
}

\begin{abstract}
Background: Heterotopic pregnancy is a rare complication of in vitro fertilization (IVF) and diagnosis and treatment can be challenging, particularly with unusual ectopic sites and desire to maintain the intrauterine pregnancy.

Case: We review the case of a 29-year-old female who presented with a cornual heterotopic pregnancy following IVF treated with exploratory laparotomy and ultrasound guided resection of the cornual pregnancy, resulting in an ongoing viable singleton intrauterine pregnancy.
\end{abstract}

Conclusion: The management of cornual heterotopic pregnancies is limited by lack of data regarding ideal treatment although several surgical and non-surgical strategies have been described. This case uses abdominal resection of cornual ectopic pregnancy using intraoperative ultrasound as an optimal approach to remove the ectopic pregnancy without disrupting the viable intrauterine pregnancy, which was in close proximity of the cornual ectopic.

${ }^{1}$ University of lowa Hospitals and Clinics, Department of Obstetrics and Gynecology, lowa City, lowa

\section{Introduction}

Heterotopic pregnancy is the coexistence of both an intrauterine and ectopic pregnancy. It is extremely rare occurring in 1 in 30,000 spontaneously conceived pregnancies. $^{1}$ This rate has increased in recent years due to the increase in IVF pregnancies; however, it is still rare occurring in about $0.8 \%$ of IVF transfers. ${ }^{1}$ Heterotopic pregnancies can be difficult diagnoses to make leading to the increased morbidity and mortality. ${ }^{1-3}$ Symptoms are vague with abdominal pain and bleeding being the most common symptoms, but with many patients remaining asymptomatic. ${ }^{2}$ Furthermore, only about half of heterotopic pregnancies are diagnosed at routine early ultrasounds. ${ }^{2}$ They can also be quite difficult to treat, especially when the pregnancy is desired and management goals include not disrupting the existing intrauterine

Please cite this paper as: Mancuso AC, Racek A, Duran EH. Abdominal resection of a cornual heterotopic pregnancy using intraoperative ultrasound: case report. Proc Obstet Gynecol. 2016;6(2): Article 4 [ 6 p.]. Available from: http://ir.uiowa.edu/pog/ Free full text article.

Corresponding author: Abigail C. Mancuso, University of lowa Hospitals and Clinics, Department of Obstetrics and Gynecology, 200 Hawkins Drive, lowa City, IA 52242. abigail-mancuso@uiowa.edu

Financial Disclosure: The authors report no conflict of interest.

Received: 21 March 2016; accepted 9 May 2016; POG in Press, 9 June 2016

Copyright: (c) 2016 Mancuso et al. This is an open-access article distributed under the terms of the Creative Commons Attribution License, which permits unrestricted use, distribution, and reproduction in any medium, provided the original author and source are credited. 
pregnancy. ${ }^{1}$ This can be further complicated when heterotopic pregnancies occur in unusual sites and while most heterotopic pregnancies occur in the fallopian tubes, $5-10 \%$ of ectopic pregnancies may be located elsewhere. ${ }^{2,4}$ Extratubal sites include the ovary, abdominal cavity, cervix, cornual region, rudimentary horn, tubal stump, and cesarean scar. ${ }^{4}$ We present a case of a heterotopic pregnancy following IVF with a cornual ectopic pregnancy in close proximity to the intrauterine gestation that was managed by surgical removal of the cornual ectopic with the aid of intraoperative ultrasound without disruption of the intrauterine pregnancy.

\section{Case Report}

A 29-year-old gravida 0 presented to our Reproductive Endocrinology and Infertility clinic for treatment with in vitro fertilization after a history of infertility of three years duration. She had a past medical history significant for endometriosis and a past surgical history significant for a laparoscopic surgery the previous year for bilateral ovarian cystectomies for endometriomas as well as right salpingectomy after chromotubation showed minimal spillage of dye from the dilated right fallopian tube.

She underwent controlled ovarian hyperstimulation, egg retrieval and subsequent fresh embryo transfer of two 8 -cell embryos on day 3 of the culture. She had a positive quantitative human chorionic gonadotropin ( $\mathrm{hCG}$ ) level of $283 \mathrm{mIU} / \mathrm{mL}$ twelve days after the transfer which was trended to 475 $\mathrm{mIU} / \mathrm{mL}(68 \%$ rise) then $943 \mathrm{mIU} / \mathrm{mL}$ (99\% rise) after two and four days respectively. She called in with some mild cramping and was scheduled for an early ultrasound. She was seen at 5 4/7 weeks of gestation and ultrasound showed a probable intrauterine gestational sac with decidual reaction and no yolk sac or fetal pole were seen. She had a corpus luteum cyst on the right ovary and multiple corpus luteal cysts on the left ovary. She was counseled about the possibility of ectopic or heterotopic pregnancy and given precautions with follow-up appointment planned in one week.

She called 6 days after this visit with moderate to severe abdominal pain, nausea, and vomiting. She went to her local emergency department and was found to have an elevated white blood cell count of 14,000 per microliter. Ultrasound showed a viable intrauterine pregnancy. She had not had a bowel movement for 10 days and pain was thought to be related to constipation so she was given an enema and discharged to home.

She continued to have abdominal pain and was counseled to come in for a clinic visit. Ultrasound at that time showed a viable intrauterine pregnancy with a fetal heart rate of 116 beats-perminute measuring 6 3/7 weeks which was consistent with dates. There was a $2 \mathrm{~cm}$ mass posterior to the uterus of unknown origin, thought possibly due to known extensive endometriosis, but heterotopic pregnancy could not be ruled out. Given her acute abdominal pain that continued despite small bowel movements, she was admitted for observation and pain control. Differential diagnosis for pain included severe constipation, appendicitis, heterotopic 
pregnancy, infected endometrioma, and tuboovarian abscess. Ultrasound for appendicitis was equivocal given large stool burden and BMI of $33 \mathrm{mg} / \mathrm{m} 2$. She was put on a strict bowel regimen and watched overnight. Her abdominal pain seemed to improve after few more bowel movements and she was otherwise stable and able to tolerate oral intake and was discharged to home on hospital day three.

She returned to clinic at $71 / 7$ weeks and at that time denied any pain or vaginal bleeding. She had a TVUS that again showed a viable intrauterine pregnancy consistent with dates and a $23 \times 20 \times 24 \mathrm{~mm}$ mass in the right cornual region consistent with possible interstitial heterotopic pregnancy that was not previously noted. Given the benign clinical presentation without pain or vaginal bleeding decision was made to follow up in 48 hours and again ectopic precautions were given. She was seen two days later and clear cornual ectopic pregnancy was noted measuring $27 \quad x \quad 22 \quad \mathrm{~mm}$ without embryonic pole or definite yolk sac (Figure 1). Patient was counseled on management options, including expectant management versus surgical management and patient opted for resection of cornual pregnancy. Given the close proximity of the intrauterine pregnancy, decision was made for surgical resection by laparotomy to allow for more precise resection of the ectopic pregnancy.

She was taken that day to the operating room where she underwent spinal anesthesia. She was placed in supine position and Pfannenstiel incision was made. The peritoneal cavity was entered and a medium Alexis retractor was placed in the abdomen and the uterus and fallopian tubes were visualized. Intraoperative ultrasound was used with a sterile transabdominal probe placed directly on the uterus to confirm the best plane to place a purse string suture around the right cornua, without interrupting the intrauterine gestational sac or placenta. A suture of 0 Chromic was used to make a pursestring suture around the base of the bulge of the cornual pregnancy. This was tied securely separating the cornual pregnancy from the remaining uterine cavity. Cautery was used to remove the top portion of the uterine cornua in a circumferential manner and contents delivered both spontaneously and with the aid of hydrodissection. The contents were grossly consistent with placental tissue and they were sent to pathology. Several figure-of-eight sutures were made overlying the cornual incision and good hemostasis was achieved. Repeat ultrasound at the conclusion of the case showed a viable intrauterine pregnancy.

The patient was admitted to the inpatient ward following surgery and recovered without complications. By postoperative day two she was meeting all postoperative goals and was discharged to home. Transvaginal ultrasound prior to discharge again showed a viable intrauterine pregnancy. She was followed in clinic at $83 / 7$ weeks and was doing well with viable intrauterine pregnancy confirmed by ultrasound and well-resolved cornual region with a hyperechoic area. Follow up again at $136 / 7$ weeks showed a viable intrauterine pregnancy with no remaining abnormalities in the cornual area. 


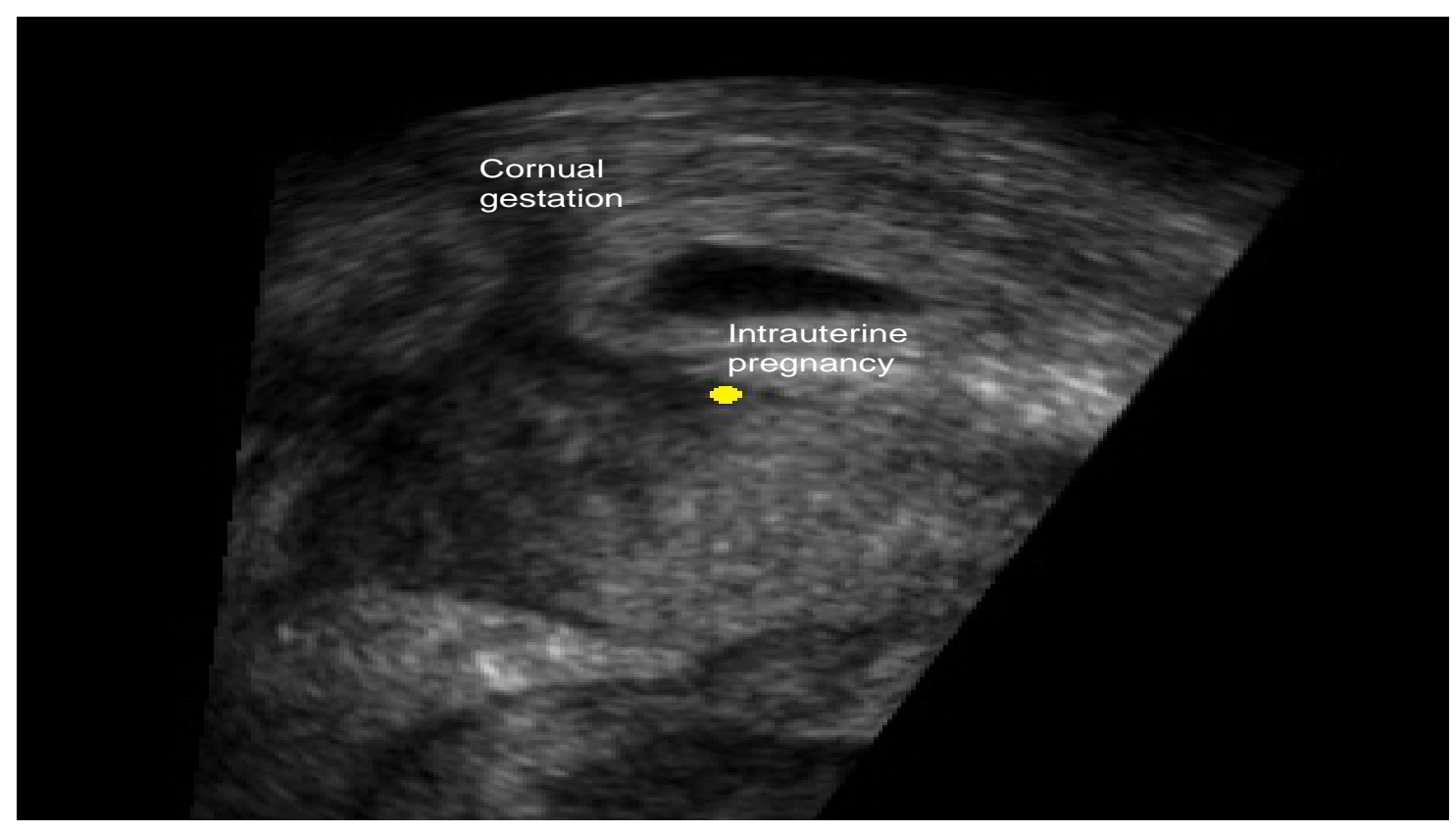

a.

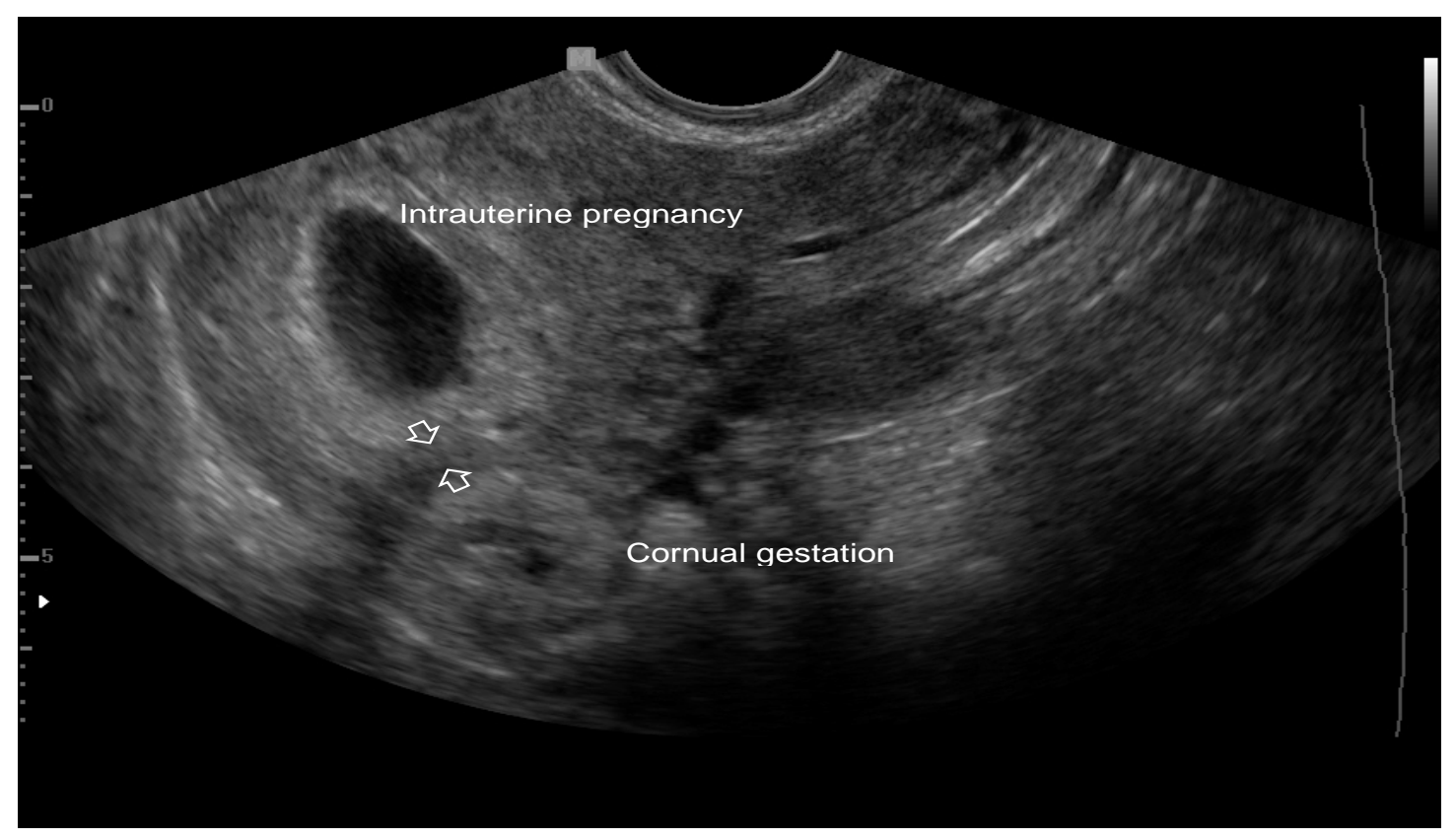

$b$.

Figure 1. Interstitial pregnancy demonstrated as an eccentric heterogeneous mass in the cornual region (a) Close proximity of intrauterine pregnancy to cornual gestation noted (b) 


\section{Discussion}

This case highlights some of the difficulties in diagnosing and treating a heterotopic pregnancy, particularly when the ectopic pregnancy is in an unusual location. If undiagnosed, cornual rupture may occur that can lead to significant morbidity, including disruption of the viable intrauterine pregnancy, as well as life-threatening hemorrhage. ${ }^{4-6}$ As seen in our case, even with a high index of suspicion it is not easy to make the diagnosis. Symptoms are usually vague with pain being the most commonly reported symptom of heterotopic pregnancy. Further evaluation is warranted in women with risk factors and repetitive/persistent symptoms. ${ }^{5}$ The viable intrauterine pregnancy seen on ultrasound may falsely reassure providers and pain may be falsely attributed to other etiologies such as constipation, appendicitis, peptic ulcer disease, and so forth. ${ }^{3}$ Vaginal bleeding may also be a less common symptom of heterotopic pregnancy compared to a typical ectopic pregnancy possibly due to the coexisting intrauterine pregnancy. ${ }^{7} \quad$ Beta-hCG levels demonstrate different dynamics in a heterotopic pregnancy. ${ }^{1,3}$ Careful ultrasound examination should be undertaken and repeated as necessary, if more than one embryo is transferred even if an intrauterine pregnancy is seen. ${ }^{6}$ On ultrasound, cornual pregnancies may be seen as a gestational sac or an eccentric heterogenous mass in the cornual region. ${ }^{8}$ An echogenic line extending into the center of the interstitial gestational sac or a thin myometrium of $<5 \mathrm{~mm}$ surrounding the gestational sac may also be seen. ${ }^{8}$
The majority of heterotopic pregnancies are treated surgically with both laparoscopic and abdominal approaches having been described. ${ }^{2,7,9}$ Other nonsurgical options include injection of Methotrexate or Potassium Chloride into the ectopic site ${ }^{2}$ or by puncture and aspiration of fetal heart of cornual pregnancy under transvaginal ultrasound guidance 4-6 weeks after the embryo transfer. ${ }^{6}$

While there have been several case reports advocating the use of laparoscopy as a minimally invasive approach for management of cornual pregnancies, we felt that laparotomy was better suited in this case due to the close proximity of the gestational sacs. Not only did an abdominal approach allow for more precise resection, it also allowed for the use of intraoperative ultrasound directly on the uterus to guide the suture plane, which is unique to this case. This case highlights not only the importance of frequent follow up when heterotopic pregnancy is suspected but not yet confirmed; it also describes the use of ultrasound as a valuable intraoperative tool for precise resection of an ectopic pregnancy that is in a close proximity to a highly desired intrauterine pregnancy.

\section{Conclusion}

Cornual heterotopic pregnancy is a rare complication to IVF and patients with vague complaints of pain or bleeding following pregnancy after IVF should be followed closely. The use of intraoperative ultrasound should be considered to guide suture lines during surgical removal of the corneal ectopic pregnancy with the goal of not disrupting the viable intrauterine pregnancy. 


\section{References}

1. Refaat B, Dalton E, Ledger WL. Ectopic pregnancy secondary to in vitro fertilisation-embryo transfer: pathogenic mechanisms and management strategies. Reprod Biol Endocrinol. 2015 Apr 12;13:30. http://dx.doi.org/10.1186/s12958-0150025-0 PubMed PMID: 25884617; PubMed Central PMCID: PMC4403912.

2. $Y u$ Y, Xu W, Xie Z, Huang Q, Li S. Management and outcome of 25 heterotopic pregnancies in Zhejiang, China. Eur J Obstet Gynecol Reprod Biol. $2014 \quad$ Sep;180:157-61. http://dx.doi.org/10.1016/j.ejogrb.2014.0 4.046 Epub 2014 May 20. PubMed PMID: 25012396.

3. Wang LL, Chen X, Ye DS, Liu YD, He YX, Guo W, Chen SL. Misdiagnosis and delayed diagnosis for ectopic and heterotopic pregnancies after in vitro fertilization and embryo transfer. J Huazhong Univ Sci Technolog Med Sci. 2014 Feb;34(1):103-7. http://dx.doi.org/10.1007/s11596-0141239-7 Epub 2014 Feb 6. PubMed PMID: 24496687.

4. Shan $\mathrm{N}$, Dong $\mathrm{D}$, Deng $\mathrm{W}, \mathrm{Fu} \mathrm{Y}$. Unusual ectopic pregnancies: a retrospective analysis of 65 cases. J Obstet Gynaecol Res. 2014 Jan;40(1):147-54.

http://dx.doi.org/10.1111/jog.12146

Epub 2013 Sep 5. PubMed PMID: 24033915.

5. Paradise C, Carlan SJ, Holloman C. Spontaneous uterine cornual rupture at 26 weeks' gestation in an interstitial heterotopic pregnancy following in vitro fertilization. J Clin Ultrasound. 2016 Jun;44(5):322-325. http://dx.doi.org/10.1002/jcu.22322 Epub 2015 Dec 16. PubMed PMID: 26677169.
6. Qiong Z, Yanping L, Deep JP, Lin Z. Treatment of cornual heterotopic pregnancy via selective reduction without feticide drug. J Minim Invasive Gynecol. 2011 Nov-Dec;18(6):766-8. http://dx.doi.org/10.1016/j.jmig.2011.08. 007 Epub 2011 Sep 25. Erratum in: J Minim Invasive Gynecol. 2012 Jan;19(1):137. PubMed PMID: 21945745.

7. Sherer DM, Scibetta JJ, Sanko SR. Heterotopic quadruplet gestation with laparoscopic resection of ruptured interstitial pregnancy and subsequent successful outcome of triplets. Am J Obstet Gynecol. 1995 Jan;172(1 Pt 1):216-7.

http://dx.doi.org/10.1016/00029378(95)90119-1 PubMed PMID: 7847541.

8. Ackerman TE, Levi CS, Dashefsky SM, Holt SC, Lindsay DJ. Interstitial line: sonographic finding in interstitial (cornual) ectopic pregnancy. Radiology. 1993 Oct;189(1):83-7. http://dx.doi.org/10.1148/radiology.189.1 .8372223 PubMed PMID: 8372223.

9. Kwon YS, Lee SH, Im KS, Ro JH. Laparoscopic Management of Heterotopic Interstitial Pregnancy with Subsequent Term Delivery. Int J Fertil Steril. 2015 Jul-Sep;9(2):265-7. Epub 2015 Jul 27. PubMed PMID: 26246887; PubMed Central PMCID: PMC4518497. 